\title{
Spectrum of histopathological lesions in the fallopian tubes
}

\author{
Gon $\mathrm{S}^{1}$, Basu $\mathrm{A}^{1}$, Majumdar $\mathrm{B}^{2}$, Das $\mathrm{TK}^{2}$, Sengupta $\mathrm{M}^{3}$, Ghosh $\mathrm{D}^{3}$ \\ ${ }^{\prime}$ Department of Pathology, ESI-PGIMSR, Kolkata, India. \\ ${ }^{2}$ Department of Pathology, R. G.Kar Medical College, Kolkota, India \\ ${ }^{3}$ Department of Obstretics \& Gynaecology, R G Kar Medical College \& Hospital, Kolkota, India
}

\section{Keywords:}

Fallopian tubes; Histopathology

\begin{abstract}
Background: Fallopian tubes are common surgical specimen in the pathology laboratory; still there is a lack of data to describe the frequency of various histological findings. The aim and objectives of this study was to describe the various histopathological findings of fallopian tubes.

Materials and Methods: Two thousand five hundred and seventy five cases where fallopian tubes were removed either separately or along with other female genital tract organs were studied retrospectively and their histopathological findings documented.

Results: Ectopic pregnancy comprised maximum number of cases closely followed by salpingitis. Primary neoplastic lesions were rare as compared to secondary malignancies. Serial sections of fallopian tube and sections from representative areas are essential for a pathologist so that the diagnosis of these pathological entities is not missed.
\end{abstract}

Conclusion: Though the fallopian tubes remain unremarkable in majority of the surgical pathological specimens, it must be subjected for histopathological examination to demonstrate the pathological lesions.

\section{INTRODUCTION}

The fallopian tubes are complex structures that represent more than conduits from ovary to endometrial cavity. ${ }^{1}$ It is a common specimen in a pathology laboratory and may be examined either alone as a salpingectomy or tubal ligation specimen, or as part of a more complex specimen from a hysterectomy and/or oophorectomy operation. ${ }^{2}$ There is a lack of data to describe the frequency of various histological findings in a general surgical pathology practice and only a few attempts have been made to publish the data in the literature.

\section{Correspondence:}

Dr Adelene Basu, MD

Department of Pathology, ESI-PGIMSR, Kolkata, India.

Email: adelene1951@gmail.com
The aim and objectives of this study was to describe the various histopathological findings of fallopian tubes surgically removed as part of total abdominal hysterectomy with or without bilateral salpingo-ophorectomy, tubal ligation with or without medical termination of pregnancy, unilateral salpingectomy and saipingo-ophorectomy.

\section{MATERIALS AND METHODS}

The data was collected retrospectively from the department of Pathology, between periods of five years i.e from July 2003 to June 2008. All the specimen of Salpingectomy either done for TAH with BSO, Ligation with / without MTP, unilateral Salpingectomy or Salpingoophorectomy was included in the study. Routinely, at least 2 sections were taken from the fallopian tube if gross pathology is 
evident; otherwise if the fallopian tube is apparently within normal limits, only one section is taken for processing. Haematoxylin \& Eosin stained slides were studied by at least two pathologists in abnormal cases. Paratubal cysts and microscopic examination for tubal metaplasia and embryologic remnants were excluded from the study.

\section{RESULTS}

Over a period of 5 years, a total of 28,455 specimens were received in the department of Pathology including fallopian tubes from 2575 patients so accounting for $9.04 \%$ of all surgically removed specimens. Total number of fallopian tubes was 4762 from 2575 patients who have undergone various surgical procedures. Fallopian tubes were part of $\mathrm{TAH}$ with $\mathrm{BSO}$, unilateral salpingectomy, unilateral salpingo-ophorectomy, tubal ligation without MTP and tubal ligation with MTP in $76.11 \%, 14.6 \%, 5.08 \%, 3.8 \%$ and $1.36 \%$ cases respectively. (Table 1 )

The maximum number of patients belonged to 36-45 years of age group ( $46.2 \%$ ) closely followed by $26-35$ years of age group. The youngest patient was a 14 years female where unilateral salpingo-ophorectomy was done for ectopic pregnancy and the eldest was 83 years old who underwent Warthin's Operation for squamous cell carcinoma of cervix.

The fallopian tubes revealed various histopathological findings which were divided into salpingitis, endometriosis, tuberculosis, hydrosalpinx, haematosalpinx and ectopic tubal gestation. Maximum number of fallopian tubes $(69.00 \%)$ were within normal limits and most of them were part of TAH with BSO specimen. Ectopic tubal gestation constituted $13.5 \%$ cases followed by salpingitis in $12.66 \%$ cases. (Table 2)

Out of 350 cases of ectopic gestation, right tube was involved in 70 cases, left in 62 cases and side along with site was not mentioned in 218 cases. However, in 56 cases which were clinically and radiologically diagnosed as ectopic tubal gestation, histopathological diagnosis of ectopic gestation could not be established due to lack of decidual reaction and absence of trophoblastic cells. In these cases, a diagnosis of chronic non specific salpingitis was given and all the cases were part of ruptured ectopic pregnancy.

Salpingitis was found in 326 fallopian tubes, with maximum cases showing involvement of bilateral tubes. Maximum cases were of chronic nature with only 15 cases of acute salpingitis. Chronic salpingitis included 3 cases of salpingitis isthmica nodosa. Endometriosis (fig.1), tuberculosis, hydrosalpinx and haematosalpinx constituted a small percentage of cases. The diagnosis of endometriosis was established when endometrial glands with endometrial stroma was found embedded within the fallopian tube wall. In all the cases of tuberculosis (fig.2), well formed epithelioid cell granulomas with caseous necrosis were found. Plical adhesions and tubal fibrosis was also noted in these cases. Endometrial tuberculosis was present in all of the cases. Hydrosalpinx and haematosalpinx constituted minority of cases.

Neoplasm of the fallopian tubes was rarely encountered with only 1 case of adenomatoid tumour (fig.3) and 1 case of primary adenocarcinoma of fallopian tube (fig.4). However, metastatic deposits were found in 5 cases, where primary was from gastric adenocarcinoma (1 case), ovarian carcinoma ( 2 cases), endometrial carcinoma (1 case) and cervical squamous cell carcinoma ( 1 case).

\section{DISCUSSION}

The histologic findings in the fallopian tubes have been described only sporadically in the literature. As this is a common specimen in surgical pathology, knowledge of general frequency of these variations can be valuable to the pathologist who identifies a potentially unusual histologic findings. ${ }^{2}$

The fallopian tubes were within normal limits in $69.00 \%$ of the cases while the rest revealed some tubal pathological lesion. Bagwan et al. ${ }^{3}$ found $33.48 \%$ of tubes involved by pathological lesions where inflammatory condition (18.05\%) of the tube constituted the major group. However, the most common histopathological finding in the present study is ectopic tubal gestation followed by chronic salpingitis. This may be because of the fact that more number of cases has been included in this study, and also, gynecologists

Table 1: Distribution of cases according to the type of surgery performed

\begin{tabular}{lccccccc}
\hline \multicolumn{1}{c}{ SPECIMENS } & $\mathbf{2 0 0 3}$ & $\mathbf{2 0 0 4}$ & $\mathbf{2 0 0 5}$ & $\mathbf{2 0 0 6}$ & $\mathbf{2 0 0 7}$ & $\mathbf{2 0 0 8}$ & TOTAL \\
\hline TAH-BSO & 182 & 390 & 358 & 426 & 315 & 289 & 1960 \\
ULSO & $\mathrm{O} 7$ & 22 & 24 & 28 & 36 & 14 & 151 \\
ULS & 17 & 59 & 73 & 88 & 87 & 52 & 376 \\
TUBAL LIGATION & 03 & 11 & 07 & 20 & 31 & 26 & 98 \\
MTP-LIGATION & 00 & 00 & 06 & 12 & 11 & 06 & 35 \\
\hline \multicolumn{1}{c}{ TOTAL } & 209 & 485 & 470 & 565 & 476 & 370 & 2575 \\
\hline
\end{tabular}

TAH-BSO:Total abdominal hysterectomy with bilateral salpingoophorectomy, ULSO: Unilateral salpingoophorectomy, ULS: Unilateral salpingoophorectomy 
enthusiastically send all the specimen of ectopic pregnancy for histopathological examination.

Ectopic pregnancy is a dangerous and potentially lifethreatening condition and as many as $95 \%$ of all ectopic pregnancies occur within the fallopian tubes. ${ }^{4}$ Hunt et al. ${ }^{2}$ found ectopic decidua in 3\% specimens of postpartum patients present in both subserosal and plical areas. Decidual reaction in both these sites along with presence of chorionic villi was seen in this study also in 350 out of 406 cases while in rest of the 56 cases, diagnosis of ectopic gestation could not be established due to lack of decidual reaction and absence of trophoblastic cells. Kirichenko et al. ${ }^{5}$ analyzed fallopian pregnancy morphologically and immunohistochemically, and found that decidual reaction was not developed in majority of cases which could be the reason of limitless invasion of cytotrophoblast and hemosalpinx development which is in accordance with the present study.

Salpingitis is inflammation of the fallopian tubes, most commonly caused by sexually transmitted micro-organisms in adolescent and adult women. It is rarely found in sexually inactive girls and generally the result of a blood-borne or genitourinary infection. ${ }^{6}$ Infertility and an increased chance of ectopic pregnancy are the most important long term complications of salpingitis. The rate of infertility is approximately $15 \%$ after a first episode of salpingitis and increases to $50 \%$ after a third episode. ${ }^{7,8}$ We found evidence of chronic non specific salpingitis in 56 cases of clinically diagnosed ruptured ectopic tubal gestation where diagnosis could not be established histopathologically.
Endometriosis is defined as the presence of ectopic endometrial tissue outside the lining of the uterine cavity and is fairly common in childbearing women. Endometriosis frequently involves the tubes in the form of nodules located in the wall or serosa. ${ }^{9}$ Microscopically, foci of endometriosis was found located within the wall of the tubes in maximum number of cases in this study.

Tuberculosis of the fallopian tube develops commonly by hematogenous spread of the organism, usually from a primary pulmonary infection and rarely by direct extension from adjacent organs or lymphatic spread from intestinal tuberculosis. Agarwal et al10 did a clinic-pathologic study of female genital tuberculosis and found the incidence declining from $1.8 \%$ in 1974 to $0.8 \%$ in 1989 and onwards. They noted the involvement of endometrium in $99.5 \%$, fallopian tubes in $94.7 \%$, cervix in $81.5 \%$, ovaries in $62.5 \%$ and vagina in $0.2 \%$ cases. All cases $(100 \%)$ of fallopian tube tuberculosis had concomitant involvement of endometrium in our study. It may be attributed to the declining incidence of genital tract tuberculosis, late presentation of the patient or less number of tubercular salpingitis patients studied in the present study.

Primary tubal neoplasms are rare and are usually only recognised at surgery or on pathological examination of the excised specimen. ${ }^{3}$ Primary fallopian tube adenocarcinoma accounts for $1 \%$ of all female genital tract malignancies. ${ }^{11}$ only one case of primary adenocarcinoma was seen in this study accounting for $0.03 \%$ of all the specimens. Majority of the patients with primary adenocarcinoma of fallopian tube present with stage 1 disease at diagnosis

Table 2: Distribution of tubal lesions diagnosed histopathologically

\begin{tabular}{|c|c|c|c|}
\hline Sr No. & TUBAL LESIONS & NO. OF CASES & INCIDENCE \% \\
\hline A & Normal & 1777 & $69.00 \%$ \\
\hline B & Abnormal & & \\
\hline \multirow[t]{5}{*}{1} & Salpingitis & 326 & $12.66 \%$ \\
\hline & - Acute & 15 & \\
\hline & - chronic & 308 & \\
\hline & - salpingitis isthmica nodosa & 3 & \\
\hline & - chronic non specific salpingitis in clinical ectopic pregnancy & 56 & $2.17 \%$ \\
\hline 2 & Tuberculosis & 5 & $0.19 \%$ \\
\hline 3 & Hydrosalpinx & 18 & $0.69 \%$ \\
\hline 4 & Haematosalpinx & 22 & $0.85 \%$ \\
\hline 5 & Ectopic tubal gestation & 350 & $13.5 \%$ \\
\hline \multirow[t]{4}{*}{6} & Endometriosis & 14 & $0.54 \%$ \\
\hline & Tumours & & \\
\hline & - Benign & 1 & $0.03 \%$ \\
\hline & - Malignant & 6 & $0.23 \%$ \\
\hline TOTAL & & 2575 & $100 \%$ \\
\hline
\end{tabular}




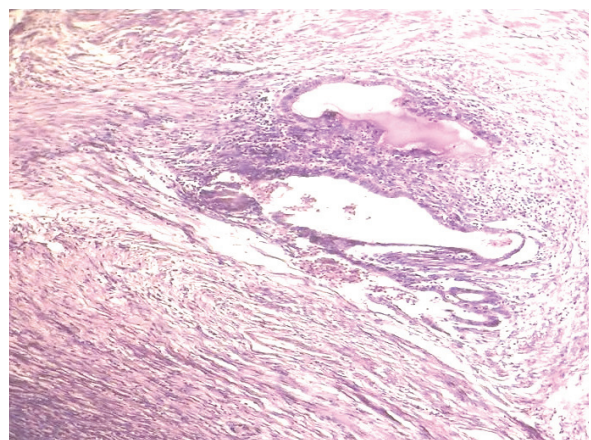

Figure 1: Endometrial glands with surrounding stroma within the fallopian tube wall (HE Stain, X100).

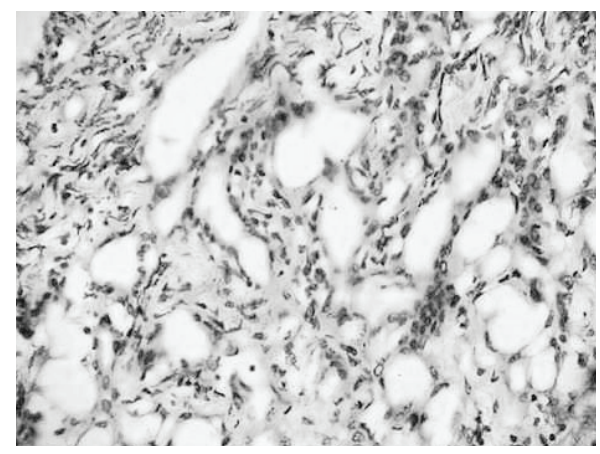

Figure 3: Conglomerate of cystically dilated spaces lined by benign flattened to cuboidal cells consistent with Adenomatoid tumour. (X400).

but their survival probability is low compared with other early stage gynecological malignancies. ${ }^{12}$ Longacre et al. ${ }^{13}$ attributed low incidence of primary malignancy in part due to admittedly arbitrary definitional criteria as it is difficult to distinguish primary tubal carcinoma from primary ovarian or primary endometrial carcinoma in patients with high stage disease. It is essential for the pathologist to section the fallopian tubes serially and submit all of the tissue for microscopic examination so that the diagnosis of carcinoma is not missed. ${ }^{14}$

In comparison, metastatic tumours were more commonly encountered ( 5 cases) in the specimen with primary from ovarian (2 cases), endometrial (1 case), gastric adenocarcinoma (1 case) and squamous cell carcinoma cervix ( 1 case). Hunt et al. ${ }^{2}$ noted metastatic carcinoma in $1.4 \%$ of fallopian tubes with tumour cell deposits as serosal implants, intraluminal tumour or infiltrating the plicae and muscularis propria. Most of the examples represent spread of an ovarian or endometrial adenoarcinoma along the peritoneal surface or through the lumen. Metastases to the tube usually come from ovarian adenocarcinoma followed by endometrial or cervical adenocarcinoma. ${ }^{15}$ Tubal metastases usually indicate poor prognosis regardless of the site of primary tumour. ${ }^{16}$

In contrast to malignant tumours, benign tumours of the fallopian tubes are rare, most frequent of which is

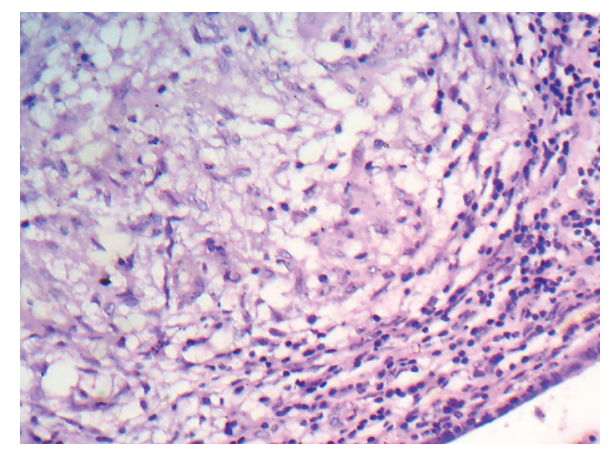

Figure 2: Well defined epithelioid cell granulomas with Langhans' giant cells and caseous necrosis. Note the fibrosis and plical adhesions. (HE Stain, X100).

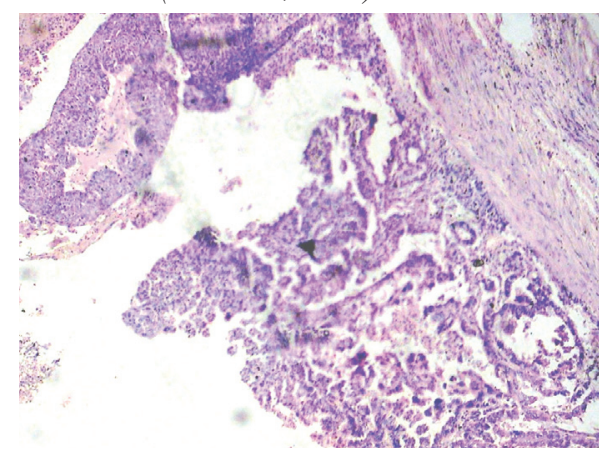

Figure 4: Primary Adenocarcinoma of the fallopian tube. Note the pleomorphic lining epithelial cells. (HE Stain, X400).

adenomatoid tumour. It is usually an incidental finding as also in present study. Its main interest to gynecologist lies in the fact that it can be mistaken for malignancy if the clinician is not aware and histopathology is the main stay to confirm the diagnosis.

\section{CONCLUSION}

Though the fallopian tubes remain unremarkable in majority of the surgical pathological specimens, it must be subjected for histopathological examination to demonstrate the pathological lesions and also, it is essential for the pathologist to section the fallopian tubes serially and submit all of the representative tissue for microscopic examination so that the diagnosis of these pathological entities is not missed.

\section{REFERENCES}

1. Anderson MC. The fallopian tube. In: Symmers WSC ed. Systemic Pathology: Female Reproductive System. Churchill Livingstone: London; 1991.pp241-61.

2. Jennifer L. Hunt, Amy A. Lynn. Histologic Features of Surgically Removed Fallopian Tubes. Archives of Pathology and Laboratory Medicine 2002;126:951-5.

3. Bagwan IN, Harke AB, Malpani MR, Deshmukh SD. Histopathological study of spectrum of lesions found in the fallopian tube. J Obstet Gynecol Ind 2004; 54:379-82.

4. Patel MD. "Rule out ectopic": asking the right questions, getting the 
right answers. Ultrasound Q 2006;22:87-100.

5. Kirichenko AK, Milovanov AP. Structural characteristic of tubal pregnancy. Arkh Patol 2004;66:27-30.

6. Van der Putten ME, Engel M, Van Well GTHJ. Salpingitis. A rare cause of acute abdomen in a sexually inactive girl: a case report. The Netherlands Cases Journal 2008, 1:326doi:10.1186/1757-1626-1326.

7. Brown-Harrison M, Christenson J, Harrison A, Matlak M. Group A Streptococcal Salpingitis in a Prepubertal Girl. Clin Pediatr $1995 ; 34: 556-8$

8. Algren S, Strickland J. Beta Hemolytic Streptococcus Group F Causing Pelvic Inflammatory Disease in a 14 -Year-Old Girl. J Pediatr Adolesc Gynecol 2005;18:117-9.

9. Sheldon RS, Wilson RB, Dockerty MB. Serosal endometriosis of fallopian tubes. Am J Obstet Gynecol 1967;99:882-4.

10. Agarwal J, Gupta JK. Female genital tuberculosis- a retrospective clinic-pathologic study of 501 cases. Indian J Pathol Microbiol 1993;36:389-97.
11. Ajithkumar TV, Minimole AL, John MM, Ashokkumar OS. Primary fallopian tube carcinoma. Obstet Gynecol Surv 2005;60:247-52.

12. Obermair A, Taylor KH, Janda M, Nicklin JL, Crandon AJ. The primary fallopian carcinoma: the Queensland experience. Int $\mathrm{J}$ Gynecol Cancer 2001;11:69-72.

13. Longacre TV, Oliva E, Soslow RA. Association of directors of Anatomic and surgical pathology. RECOMMENDATIONS for the reporting of fallopian tube neoplasms. Hum Pathol 2007;38:1160-3.

14. Colgan PJ. Challenges in the early diagnosis and staging of fallopian tube carcinomas associated with BRCA mutations. Int J Gynecol Pathol 2003;22:109-20.

15. Gompel C, Silverberg SG. Pathology in Gynecology and Obstetrics, 3rd ed. JB Lippincott Company: Philadelphia;1985. 278pp.

16. Mazur MT, Hsueh S, Gersell DJ. Metastases to the female genital tract: Analysis of 325 cases. Cancer 1984;53:1978-84. 